\title{
Consistency in Ground Potential Rise Estimation Utilizing Fall of Potential Method Data
}

\author{
Darren J. Woodhouse and Richard H. Middleton, Fellow, IEEE
}

\begin{abstract}
Analyzing Fall of Potential (FOP) test data from a grounding system current injection test is the prevalent method used to determine Ground Potential Rise (GPR). Achievable tolerance in analyzing near ideal FOP results using recognized and alternative methods were assessed previously, whereas this paper investigates distortions by buried metallic systems, such as leaky cables and pipelines, based on both simulated and field data. To work correctly with "real" data, pre-conditioning FOP data may be necessary; removing "outliers" due to real world "pathologies" in the earthing system. Applying several "cost" functions to the methods created by the combination of conditioning schemes and FOP response models produced a significant number of estimation methods. To determine the most consistent estimation methods a methodology was developed which assessed all identified methods by comparing the estimates produced with the simulation result. This allowed the most consistent and accurate GPR estimation methods to be identified.
\end{abstract}

Index Terms-Current injection testing, earthing, grounding, impedance, measurements, parameter estimation, testing.

\section{INTRODUCTION}

$\mathbf{P}$ REVIOUS work by the authors [1] examined the performance of fundamental GPR estimation methods when used to analyze near ideal data and near ideal data subject to white noise. This paper details work which examines the original estimation methods and more advanced methods applied to both simulated test data and field data. The simulated test data was generated from finite element models of earthing systems with various external buried metallic systems to introduce more realistic response distortion [2].

The fall of potential method [3] uses the response of the soil surrounding a grounding system leaking current to determine the grounding system's GPR. Executing the fall of potential method requires the potential of the soil response to be measured with respect to the GPR at increasing distances from the edge of the earthing system (x), as shown in Fig. 1. This is undertaken during a current injection test described in [4] and [5]. Many utilities then use a classical GPR estimation technique, based on a point source model, which graphically extrapolates the soil profile to remote earth.

Analysis of simulated field data using the methods outlined in [1], including the equivalent of the classic estimation tech-

Manuscript received June 19, 2003; revised January 11, 2004. Paper no. TPWRD-00308-2003.

D. Woodhouse is with Safearth Engineered Solutions, Wallsend, NSW 2287 (e-mail: dwoodhouse@energy.com.au).

R. H. Middleton is with the ARC Centre for Complex Dynamic Systems and Control, The University of Newcastle, Callaghan 2308, Australia (e-mail: Rick.Middleton@newcastle.edu.au)

Digital Object Identifier 10.1109/TPWRD.2004.838642
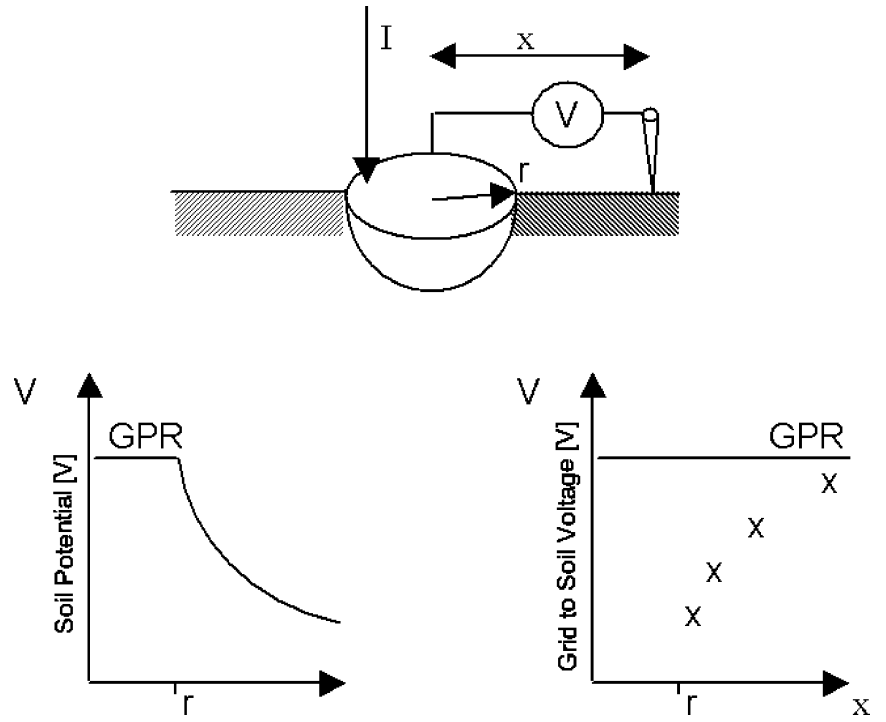

Fig. 1. Fall of potential method relationships.

nique, produced greater than anticipated estimation error. More complex methods were investigated for improved estimation capability when applied to data subject to more realistic disturbances. The importance of accurate GPR estimation was examined in a dissertation by Carman [6] where it was shown that the potential hazard risk associated with an installation due to GPR associated hazards was nonlinear. It was shown that the fibrillation risk in one instance rose by $15 \%$ due to an increase in the GPR of only 7\%. GPR estimates also establish a benchmark for an earthing system injection test as all voltage measurements are made proportional to them. Measurements such as touch and step voltages are scaled as a function of the GPR and GPR estimate error thus extends to those results. Finally, a FOP test is influenced by the environment surrounding the installation. Restrictions imposed by private property and hard surfaces complicate its execution. Any tool which simplifies its analysis or increases the flexibility or robustness in the field is of benefit to the tester.

Comparison was made of a number of practical variations in estimation method including FOP response model, cost assessment, data subset selection, combination of methods and data pre-conditioning. The number of methods available for estimating GPR when these variations are considered make manual comparison impractical. It also raises the question of whether there is any method/s which display consistent performance for a wide range of data sets and distortions.

In estimating GPR an important issue is the conservatism or otherwise of the result. A conservative result is an estimate 
greater than the actual GPR, since such an estimate will lead to an over-estimation of touch and step voltages. The desired outcome of GPR estimation then is the identification of a method which consistently produces a conservative result or where the expected result consistently lies within the tolerance range defined.

The analysis using the methods outlined may require factorial (in the number of data points) computation times and are therefore computable only for small data sets. Methods were also examined that better managed these computational difficulties to give practical numerical algorithms.

The assessment of which algorithms performed consistently included a range of simulated test cases ranging from simulated cases with no distortions and simple earthing systems to cases which include a number of distortion sources and independent yet "interfering" grounding systems.

To achieve "consistency" a number of qualities are required of the estimation technique including accuracy, robustness, repeatability, computability, and conservatism. The result of the assessment is to indicate the preferred estimation method which achieves the most "consistent" result across the range of examined cases. Of an initial set of 2304 methods (including combinations of conditioning schemes) a preliminary cull down to 22 methods, followed by simulations for 19 test cases identified several optimal methods for estimating GPR from FOP data.

\section{FundAMENTAL METHODS}

In the original paper [1], two fundamental models for FOP response, linear and offset, were outlined. These methods form the basis of all the GPR estimation methods that were examined. More recently, alternative methods and additional models for the estimation processes were introduced and the initial nomenclature was found to be inappropriate. Consequently the linear and offset models have been renamed to the "First Order Rational (FOR)" and "First Order Rational Offset (FORO)" models respectively. For clarification the fundamental methods are described in the following sections.

\section{A. First Order Rational (FOR) Model}

As the measurement distance increases, the behavior of the measured voltage can be described by modeling the earthing system as a point source in a resistive media [7], as described in (1). The following analysis forms the basis of a FOP test and its analysis, using the FOR model. The derivation assumes the soil resistivity is homogeneous, the size of the earthing system is negligible in comparison to the spacings used, and the measurement reference is the earthing system potential (GPR)

$$
V=\mathrm{GPR}-\frac{\rho I}{2 \pi x}
$$

where

$\mathrm{V}$ voltage between GPR and soil " $\mathrm{x}$ " meters from earthing system $(\mathrm{V})$;

$\rho \quad$ soil resistivity $(\Omega . \mathrm{m})$;

I fault current (A);

$\mathrm{X} \quad$ separation between earthing system and point on soil (m).

\section{B. First Order Rational Offset (FORO) Model}

The First Order Rational Model, as described by (1), is based on a point current source and uniform soil resistivity. To improve upon the estimates of the method, a new model which better describes the system was trialed [8]. The First Order Rational Offset model was based on empirical equations used for Mutual Earth Resistance (MER) and conductive coupling calculations. The FORO model takes the form described in (2)

$$
V=\operatorname{GPR}-\frac{\rho I}{2 \pi(x+r)}
$$

where $r$ is the equivalent radius of the earthing system (m).

\section{HIGHER ORDER METHODS}

The application of least squares regression to a linearized form of a system model attempts to match the model's response to a number of fixed points. The models chosen to match to these sets in this application are simplifications of a complex physical system. The premise applied here is that with the addition of a higher order term the model is better able to match the physical response and consequently better estimates achieved. Both second and third order methods were examined as follows:

\section{A. Second Order Methods}

In this case the second order term is added to the applicable fundamental model. The resulting models are still matched to the data using linear regression, however, the selection of an optimal model, or cost, is based on the co-efficient of the additional 'quadratic' term. That is, the most desirable match is that model which has the smallest higher order component.

Applying this method to the FOR model we may describe the problem with familiar terminology by applying the following transformation to (1):

$$
\begin{aligned}
& \mathrm{y}=\mathrm{x}^{-1} \\
& \mathrm{~V}=\mathrm{GPR}-\frac{\rho I y}{2 \pi} .
\end{aligned}
$$

Collecting all the constants and the addition of a higher order term we arrive at the relationship described by (5).

$$
\mathrm{V}=\mathrm{a} \cdot \mathrm{y}^{2}+\mathrm{b} \cdot \mathrm{y}+\mathrm{c} .
$$

The use of higher order methods can be applied to either the FOR or FORO models, resulting in two methods describable as second order methods, being:

- second order rational model (SOR);

- second order rational offset model (SORO).

\section{B. Third Order Methods}

In this case a third order term is added to the applicable fundamental model with the resulting models matched as per the Second Order methods. A second order term was not included as closer analysis of the Finite Element Model of the physical system found that the physical system contained odd harmonic terms. Initial analysis using the second order methods produced results similar to the fundamental methods so by introducing a third order term it was hoped that the results would improve 
significantly. The use of cubic methods was applied to the FOR model resulting in the Third Order Rational [TOR] Model, as described in (6)

$$
\mathrm{V}=\mathrm{a} \cdot \mathrm{y}^{3}+\mathrm{b} \cdot \mathrm{y}+\mathrm{c} .
$$

The remainder of the paper describes the investigation of the new GPR estimation methods.

\section{LEAST SQUARES TRANSFORMATIONS}

Even though a model has been derived to simulate the FOP response and consequently estimate the GPR, a mechanism for acquiring a "best model" is required. A common method for optimizing a given model to test data is through the use of least squares estimation [9]-[11]. In an "ideal" system a linear model can be described as

$$
\mathrm{Y}=\mathrm{X} \cdot \theta
$$

However, our systems are not ideal and are subject to noise. If we assume that the noise is noncorrelated then we can estimate the system as

$$
\mathrm{Y}^{\prime}=\mathrm{X} \cdot \theta+\eta
$$

where $\eta$ represents the noise in the system.

To estimate the parameters of the system where noise is expected the least squares minimization or linear regression given by (9) is used

$$
\theta^{\prime}=\left(\mathrm{X}^{\mathrm{T}} \cdot \mathrm{X}\right)^{-1} \cdot \mathrm{X}^{\mathrm{T}} \cdot \mathrm{Y}
$$

where $\theta^{\prime}$ represents the estimation to $\theta$.

The models outlined in (1) and (2) are not linear and prior to their use in a least squares estimation, a linearization of an equation's parameters is required. In the case of (2) this is achieved by rewriting as

$$
V=\frac{b_{1} \cdot x+b_{0}}{x+a_{0}}
$$

where $\mathrm{V}$ and $\mathrm{x}$ are measured and $\mathrm{b}_{1}, \mathrm{~b}_{0}$ and $\mathrm{a}_{0}$ are constants to be determined. Equation (10) can be rearranged as

$$
V=-a_{1}^{\prime} \cdot x \cdot V+b_{1}^{\prime} \cdot x+b_{0}^{\prime}
$$

where $a_{1}^{\prime}=\left(1 / a_{0}\right), b_{1}^{\prime}=\left(b_{1} / a_{0}\right)$, and $b_{0}^{\prime}=\left(b_{0} / a_{0}\right)$. (9).

Equation (11) can be used to calculate an estimate for $\theta$ using

\section{Weighted LEASt SQuares}

Weighted least squares (WLS) allows the effects of individual data elements to be either favored or de-emphasized within least squares analysis. As part of the analysis of FOP data, weighted least squares is used to perform outlier rejection, that is, remove those data points which do not conform to the relationship described by the bulk of the data points. This rejection can be implemented by setting the relevant weightings to zero.
To implement weighted least squares the normal equation for least squares analysis is modified by the addition of the weighting matrix $\mathrm{W}$ as given by (12)

$$
\theta^{\prime}=\left(\mathrm{X}^{\mathrm{T}} \cdot \mathrm{W} \cdot \mathrm{X}\right)^{-1} \cdot \mathrm{X}^{\mathrm{T}} \cdot \mathrm{W} \cdot \mathrm{Y} .
$$

The weightings matrix in its most primitive form is implemented as a diagonal matrix, with the nth diagonal element or weighting corresponding to the nth data point in the FOP data. Increasing a weighting's value increases its significance and influence on the parameters estimate. Each weighting was given an initial value of 1 . The association of a single ordinal value with each data point facilitates desirability of individual data points. The more complex situation of inter-point dependency describable by point weightings was not considered due to its complexity. The setting of criteria to distinguish "good" and "bad" data points allows outlier rejection to be implemented.

\section{OUTLIER REJECTION}

It was noted during the assessment of the GPR estimator's performance [1] evaluation that points lying outside that expected of an "ideal" response (i.e. an "outlier"), significantly affected the estimate. The best way to deal with these points was to attempt to reject them as outliers using weighted least squares.

As the systems being modeled are nonlinear, the weightings required to add or remove a point are nonlinear. Consequently, suitable transformations are required to implement weightings linearly so that decisions can be made about which points to reject linearly.

The FOP models when transformed to a nonlinear equation, as per (11), produce polynomials of the order of the $x y$. When these elements are rationalized into the matrix $\mathrm{X}$, the resultant matrix $\mathrm{X}^{\mathrm{T}}$. $\mathrm{X}$ has complex polynomial combinations of $\mathrm{x}$ and $\mathrm{y}$. To produce weightings which result in linear desirability, the power of the weightings will need to be proportional to the square of the most significant power in the elements of $X$.

Given that the voltage measurement at distance $\mathrm{x}$ from an earthing system is a function of the distance from the earthing system, the GPR and the size of the earthing system, the following description of the WLS process can be made. The soil potential profile will be defined by (13).

$$
\mathrm{V}(\mathrm{x})=\mathrm{f}(\mathrm{x}, \mathrm{GPR}, \mathrm{r}, \mathrm{m})
$$

where

$\mathrm{x} \quad$ Distance from earthing system (m)

GPR Ground potential rise of earthing system (V)

$\mathrm{r} \quad$ Equivalent radius of earthing system (m)

$\mathrm{m} \quad$ Gradient $\left(\mathrm{V} \cdot \mathrm{m}^{-1}\right)$

$\mathrm{V} \quad$ Soil voltage with respect to EPR (V).

The WLS process begins with $\mathrm{W}=1$ (the identity matrix), see Fig. 2. An estimate to GPR is made using (13) which produces a model and estimates for GPR, $m$ and $r$. The weightings are then recalculated using (14), for the nth data point and jth iteration. If the weightings meet the analysis's end condition the calculation halts, otherwise the process is repeated with the new weightings matrix.

$$
\mathrm{W}_{\mathrm{n}}=\left[\mathrm{y}_{\mathrm{n}}-\mathrm{f}\left(\mathrm{x}_{\mathrm{n}}, \mathrm{GPR}_{\mathrm{j}}, \mathrm{m}_{\mathrm{j}}, \mathrm{r}_{\mathrm{j}}\right)\right]^{-2} \text {. }
$$




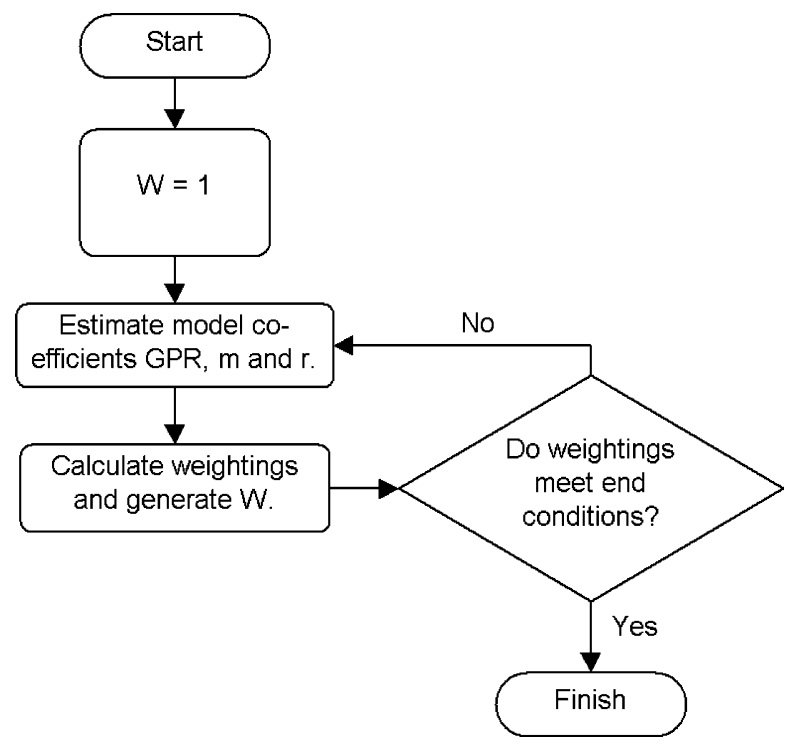

Fig. 2. Weighted least squares process.

Outlier rejection can be applied in two ways, as a conditioning to a fundamental method or used directly to make a GPR estimate. For this reason outlier rejection is described as a "semi fundamental" method. Outlier rejection can be based on either the FOR or FORO models:

- Outlier Rejection based on FOR Model [JFOR]

- Outlier Rejection based on FORO Model [JFORO]

\section{Cost Functions}

All the estimations made thus far have been based on the standard least squares cost function, as given by (15)

$$
C=\sqrt{\frac{1}{n} \sum_{i}^{n}\left(f\left(x_{i}\right)-y_{i}\right)^{2}}
$$

where

$n \quad$ number of points in FOP data set;

$x_{i} \quad$ spacing of point $i$;

$y_{i} \quad$ voltage of point $\mathrm{i}$;

f model response at $\mathrm{x}_{\mathrm{i}}$;

C cost.

This cost function is weighted equally for all points in the FOP data set. It also avoids bias toward small data sets through the application of the $1 / n$ term to the summation. The points closest to the earthing system are sensitive to factors such as earthing system geometry and soil resistivity structure. Consequently the cost of errors for points with smaller spacings should not be as significant as that determined for points at larger spacings. The cost function for the least squares analysis was consequently altered to reflect the importance of point spacing. To assess the best cost estimate a number of cost functions where analyzed of the following form:

$$
C=\sqrt{\frac{1}{n} \sum_{i}^{n}\left(f\left(x_{i}\right)-y_{i}\right)^{2} x_{i}^{r}}
$$

where $r=-1,0$ or 1 .
Just as distance should affect the cost of an estimate, so should the number of data points used to produce an estimate. The greater the number of points used to generate an estimate the greater the confidence in the estimate. To reflect this the cost function could be modified as described by (17)

$$
C=\sqrt{\frac{1}{n^{t}} \sum_{i}^{n}\left(f\left(x_{i}\right)-y_{i}\right)^{2}}
$$

where $t=0,1$ or 2 .

There is no convenient means to determine a-priori what the most appropriate value of $\mathrm{t}$ is so this was also investigated.

\section{CONDITIONING METHODS}

We use the term "conditioning methods" to denote mechanisms for ignoring a group of points in the original data set. As such, these methods condition the data before being used to calculate a GPR estimate. Two conditioning methods are presented in this paper, namely:

- points beyond knee;

- geometrically conditioned spacings.

\section{A. Points Beyond Knee}

The results of the original paper [1] found that GPR estimates based on the FOR model benefit from the reduction of the number of data points used in the FOP analysis to those points beyond the FOP response's knee. The knee of a FOP response is loosely defined, but for the analysis it is important to recognize the knee as the region where the FOP response loses its geometric dependence. The FOP response beyond the knee can then be modeled as a hemispherical electrode buried in a resistive media.

The majority of test FOP responses can be described by the regions depicted in Fig. 3 known as the "typical response." Using this typical response, an approximation method for locating the knee of the FOP response is given by the following algorithm.

1) Determine the gradient of the line defined by the origin and the last point in the response, to be referred to as the "mid-gradient."

2) Determine the point in the FOP response where the gradient of the model parallels the mid-gradient line, which defines the knee point.

3) Use the points in the FOP response located at distances beyond the knee point.

\section{B. Geometric Conditioned Spacings}

Geometric Conditioned Spacings, or Geometric Conditioning, is based on the following premises:

1) The GPR is greater than any voltage measured in the FOP data set, and

2) For increasing FOP data spacings the voltage should increase.

The "Geometric Conditioning" of FOP data requires comparison of consecutive data points. The point with the larger spacing is removed from the data set if the voltage is less than the voltage for the point with the smaller spacing. The next pair is the largest 


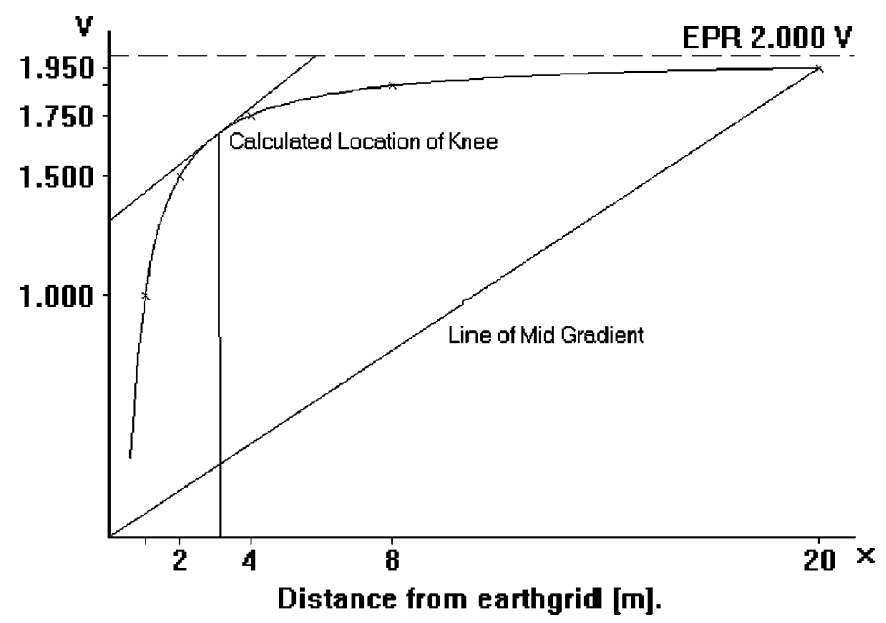

Fig. 3. Approximating the FOP response knee point.

point left from the previous pair and the next largest spacing. The process terminates when there are no more data points to consider. Geometric Conditioning was implemented as a simple method for removing points which defy the asymptotic nature of the typical FOP response. By using this method to filter the data, it was anticipated that the susceptibility of the estimators to localized distortions should be reduced.

\section{DATA SUbSET SELECTION}

Sources of distortion to a FOP response tend to affect the region of the FOP response nearest the source of the distortion. Previous data conditioning methods can remove single points or points in a specific region of the FOP response. In contrast, data subset selection allows any subset to be removed, allowing it to be used to remove the effect of these localized distortions. The simplest form of subset selection would involve a "brute force" comparison of the results of estimation with all possible subsets of data.

However, methods which examine all subsets of a FOP test data set were found to require extensive computation time. One example required a number of days to produce a result. Consequently, methods to reduce the computation time were examined.

One method to reduce the computations may be thought of as a branch and bound method. This based on the observation that the estimation methods based on the least squares method generate a cost value for a GPR estimate. This cost value can be derived by an estimation method applied to a data subset containing $\mathrm{n}$ data points as given by (15) or one of its forms. In such cases the following method was used to eliminate subset groups from consideration.

Given any subset of $\mathrm{n}$ data points the cost of that subset is given by a form of (18)

$$
J_{n}=\frac{1}{n} \cdot \sum_{j}^{n} e_{j}^{2}
$$

Any other subset that contains those $\mathrm{n}$ data points will only achieve a lower cost if the additional cost is less than the average cost per point. For a subset with an additional point to that used in (18) the cost can be recalculated as (19)

$$
J_{n+1}=\frac{1}{n+1} \cdot \sum_{j}^{n+1} e_{j}^{2} .
$$

Effect of Data Subset Selection on Estimation Time Comparison of Weightings Selection with Estimation Considering All Combinations

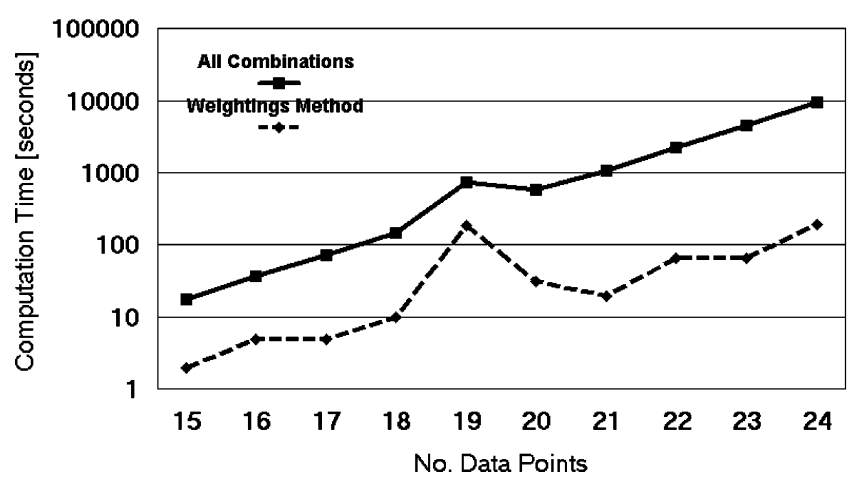

Fig. 4. Performance of "stopping criteria."

The cost ratio between the subsets can be defined by (20).

$$
\frac{J_{n+1}}{J_{n}}=\frac{\frac{1}{n+1} \cdot \sum_{j}^{n+1} e_{j}^{2}}{\frac{1}{n} \cdot \sum_{j}^{n} e_{j}^{2}} .
$$

By noting that

$$
\sum_{j}^{n+1} e_{j}^{2} \geq \sum_{j}^{n} e_{j}^{2}
$$

it can be shown that the lower bound of the ratio of the subset error is given by

$$
\frac{J_{n+1}}{J_{n}} \geq \frac{n}{n+1} .
$$

Given the error for any subset $\left(e_{s}\right)$ and the maximum number of additional points that can be added to the subset $(\mathrm{m})$ the best error achievable by any derived subset can be calculated using

$$
e_{\text {best }}=\frac{n}{n+m} \cdot e_{s} \text {. }
$$

If $e_{b e s t}$ is greater than the error of the best estimate thus far then calculating estimates for all derived subsets of the current subset can be ignored. Where different cost functions to (18) are used then a new lower bound function will need to be derived for use. This 'stopping criteria' was introduced into the estimation method used by the various methods previously established.

From the results described by Fig. 4 it can be seen that the improvement is excellent. An estimation based on 24 data points reduced from over 2.5 hours computation time to just longer than 3 minutes, approximately $2 \%$ of the time required by the complete computation. The only problem is in principle it is only applicable to methods based on a least squares type cost function.

\section{Estimation Method InVESTIGATION}

To determine which method, or group of methods, produces the most consistent, robust and accurate GPR estimate a computer program was written to examine all conceivable estimation methods. The GPR was estimated using every available variation in fundamental method $(9+1)$, semi fundamental method $(3+1)$, cost function (20) and conditioning methods (4). This potentially equates to 3200 methods to be assessed. Due to time 
considerations only the weightings method of data subset selection was used.

The number of variations in estimation method made it necessary to establish a calculation benchmark using a set of check files to rigorously and efficiently check and compare the performance of all estimation algorithms. The series of check files were based on earthing system models so the "true" GPR was known or on tested systems with a high degree of confidence in the results. All estimation methods could then be applied to each check file and the results of each estimation compared to the expected GPR.

The check files used fell into three categories. The first group was based on the earthing system FOP response models, FOR and FORO, introduced in [1]. Ideal FOP response data sets were generated using both models as ideal base cases. The ideal cases were then distorted manually from the ideal responses to produce cases including one or two outliers with varying levels of distortion. The cases produced by manual distortion included single outlier, two consecutive outliers and two spread (nonconsecutive) outliers.

The second set of check files were based on Finite Element Analysis (FEA) models of several earthing systems. These models were used to produce more realistic FOP response data sets by introducing distortions resultant from the earthing system configuration or unassociated earthing systems. These distortions were developed by modeling buried metallic systems such as counterpoise conductors and pipelines, and bonded in the following configurations:

- Direct-A buried, un-insulated metallic circuit connected to the earthing system being tested.

- Indirect-A buried, un-insulated metallic circuit not connected to the earthing system or any other earthing system involved in the test.

- Remote-A buried, un-insulated metallic circuit connected to remote earth, by an earth insulated or noninsulated circuit.

The third set of check files were based on field results where a high degree of confidence was held in the actual GPR. This was due either to a high correlation between test results and design results, numerous FOP tests or a combination thereof.

Sources of distortion not examined include variations in soil structure along the route of the FOP response, horizontal stratification of soil structure exceeding two layers and vertical soil stratification [12]. It has also been assumed that the injection test setup is such that the zones of influence of the two current electrodes can be considered to be isolated. The check files used are available for inspection at http://www.safearth.com.au/research/djw/checkindex.html.

The investigation was implemented using a suite of $\mathrm{C}++$ based applications. The first application was used to apply all the identified estimation methods, called the "Comprehensive Calculator," producing a report for each file detailing the results of the analysis. When all the cases in the set where completed the files detailing the results were parsed to rank and sort the results.

Any method which produced an invalid estimate for a test case was rejected. The estimate with the smallest error for each case, the "best method," was used as the basis of a ranking for all methods for that case. The "best method" was ranked 1, a method with twice the error of the best method was ranked 2 and so forth. The final ranking for each method was based on the inverse of its average rank for the $n$ cases examined as per (24)

$$
r_{j}=\left(\frac{1}{n} \sum_{i}^{n} m_{i j}\right)^{-1}
$$

where

$r_{j} \quad$ average rank for $\mathrm{jth}$ estimation method

$m_{i j}$ ranking for case $\mathrm{i}$ and estimation method $\mathrm{j}$.

A second method of ranking was generated using the standard deviation of each methods rankings as per (25).

$$
s_{j}=\left(\frac{\sigma_{j}}{n} \cdot \sum_{i} m_{i j}\right)^{-1}
$$

where

$s_{j} \quad$ ranking for case i estimation.

$\sigma_{j} \quad$ rank standard deviation for jth estimation method.

\section{Results}

The results of the investigation required that the investigation be iterated a number of times, to correct application errors and due to the following observed characteristics of the estimations methods:

- The more complicated the estimation method the greater the number of points required to generate an estimate.

- The preconditioning methods can remove a significant number of points, restricting the number of estimation methods that would produce a valid result.

- The combination of these two effects tended to favor the less complicated estimation methods that required fewer points.

- One aim of the investigation was to reduce the number of points required to be collected as the number of points directly effects test cost and duration.

These observations resulted in the following:

- The set of investigation files was modified so that the minimum number of points in any file was 8 . The minimum was not made higher as this went against the aim of point minimization.

- The application of geometric conditioning methods was "softened," by applying the conditioning methods strictly before any other estimation method was performed. It was also required that a minimum number of points be retained in the set so that an estimate was always produced.

The process of ranking the results required that a minimal error be identified. While it is readily acknowledged that an ideal result will have zero error, no comparison can be made with other finite results in this case. It was also identified that a result with an error of $0.001 \%$ and another result with an error of $0.1 \%$ would have roughly equal value to the end user where current error levels are anticipated to be in the order of $10 \%$. The procedure for ranking the results was thus based in the maximum of the minimum error found and the minimum error limit. By increasing the minimum error it allowed the investigation to identify methods that may not produce perfect results for the ideal 
TABLE I

IDENTIFIED SUBSETS

\begin{tabular}{l|cc} 
Set & Files & Minimum \% Error \\
\hline $\mathbf{1}$ & $1-8$ & 0.25 \\
$\mathbf{2}$ & $9-15,18-19,21$ & 2 \\
$\mathbf{3}$ & 16 & 1 \\
$\mathbf{4}$ & 17,20 & 0.7
\end{tabular}

cases in the investigation set, but could produce estimates with very low error levels for all cases. Setting the minimum error level was also a question of investigation. Thus the assessment was conducted for a range of minimal error values with the results described in Table I.

The results found that no method could achieve a score even approaching 1 even with allowable errors as high as $100 \%$, demonstrating that there is no ideal estimation method. As no single optimal estimator could be identified the results were examined further to determine if optimal estimators could be found for subsets of the investigation files. This was successful and four groups of estimators corresponding to four file subsets were identified. In identifying these subsets a minimal error had to be established for which a unit score was achieved. The identified subsets and their minimal errors are summarized in Table I.

The best methods identified for each subset using the "s" and "r" rankings were:

- For Subset 1 the top 57 ranked methods were based on SORO, pre-conditioned using outlier rejection (any model) and using any variation of least squares cost function.

- For Subset 2 the top 50 ranked methods were based on TORO, pre-conditioned using outlier rejection (JFOR), points beyond knee, geometric conditioning and using any variation of least squares cost function.

- For Subset 3 the top 180 ranked methods were based on SORO, pre-conditioned using outlier rejection (any model) and using any variation of least squares cost function.

- For Subset 4 the top 21 ranked methods were based on TORO, pre-conditioned using outlier rejection (JFORO), points beyond knee, geometric conditioning and any variation of least squares cost function.

These methods represent the best methods to use to obtain a result from the investigation set. To determine which estimate to use from the four methods as the ultimate estimate was not established. The methods identified were consequently reapplied to the investigation file set and the results examined for some trend to aid in selecting the best estimate to use. The results of this are summarized in Fig. 5 describing the relative errors with the expected result when the estimate chosen is based on the average, maximum and the lowest least squares error.

The results shown in Fig. 5 indicate that the best selection method to use is to choose the maximum result, except in the case of file 16. This produces a conservative result and a lower error than the estimate chosen using the average result. The exceptional result for file 16 is due to the inverted nature of the response. This response is completely contrary to that expected

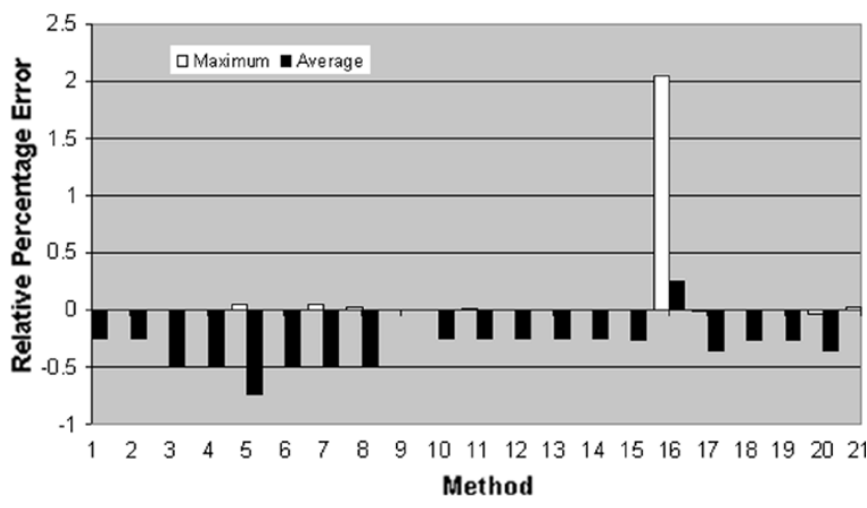

Fig. 5. Error of estimates selected using maximum and average results.

by any of the models used here. To overcome this would require either additional modeling of localized disturbances or the removal of the disturbance from the data by the user. Removal of the disturbance by the user requires some experience on the user's part, sufficient data to verify the existence and nature of the disturbance, and possible identification of the source of the disturbance in the field.

\section{CASE Studies}

The installation chosen for the case study was injection tested in 1993, well before the requirements of this assessment were anticipated so test results could not be biased. It would have been preferable to assess the performance of the selected estimation method (SEM) by applying it to a published case for GPR estimation but to our knowledge no such case exists.

The site had the following characteristics, making it suitable for the purposes of this assessment

- It is located in an extensive inland plain with a consistent soil structure, verified by soil resistivity tests performed around the period of the injection test, and by the published geological data [13]. Soil resistivity tests performed at locations up to $100 \mathrm{~km}$ from the site found soil resistivity structures consistent with a homogeneous $65 \mathrm{ohm} . \mathrm{m}$ soil model.

- The documentation for the "as built" earth grid was available and no alteration had been made to the installation between commissioning and testing.

- The installation had no significant interconnections to other earthing systems. The LV system it supplied was an overhead system, with only short underground cable sections used to connection to the distribution network. The HV network was also an overhead network with OHEW's only installed on a few spans out of the installation.

- A distortion was evident in the FOP results which was due to the test path crossing an underground from the installation with poor outer insulation and an earthed sheath.

- The raw FOP test results are available at http://www.safearth.com/research/djw/casestudy.html.

A model of the earthing system was created based on the information available on the earthing system, the soil resistivity and the test injection level using FEA software. The initial assessment of the installation performed in 1993 reported excellent correlation $(<1 \%$ difference) between the earth grid resis- 




Fig. 6. Original FOP test data compared with model response.

tance predicted by the FEA model and the grid resistance as tested based on the method available at that time for analyzing FOP test results.

The results of the FOP test and the soil response predicted by the model are shown in Fig. 6, showing a good alignment between the expected result and the predicted response. It also highlights the distortion in the FOP response due to the underground cable. The test current of $3 \mathrm{~A}$ was used so that the resultant voltages are kept within safe limits for test personnel. Actual fault levels for the installation are 1000 times larger than the test currents so expected hazard levels are of the order of $1000 \mathrm{~V}$.

Re-examination of the original soil resistivity test results found that there existed a thin high resistivity layer atop the otherwise homogeneous soil strata. This high resistivity surface layer is created by the dry humid weather typical of the area. Its thickness is dependent on whether the surface is bare soil or has a covering such as grass, concrete or asphalt. The initial results then are based on the upper layer being absent. The effects of its presence were, however, modeled using the FEA model and showed that the grid resistance could rise by up to $10 \%$ and the soil potential gradients become steeper in proximity to the installation.

The analysis of the FOP data was undertaken using the SEM. A subset of the FOP data set was also analyzed created by removing the points in the vicinity of the disturbance and with more even spacings (see Fig. 7). The results of the analysis are summarized in Table II. The expected GPR in this case was 673 $\mathrm{mV}$. The original investigation of this installation determined the GPR to be $620 \mathrm{mV}$ (-8\% error) based, as is the traditional method [14], on a FOR model of the last points in the data set.

From Table II it is clear that the original data set provides a conservative answer with a large error. The modified data set with a significantly smaller number of points produces a better, slightly nonconservative estimate. If the ground grid condition and the accuracy of the soil resistivity results are considered, being parameters to the FEA model, it can be shown that the modified estimate result lies within the tolerance of the FEA model.

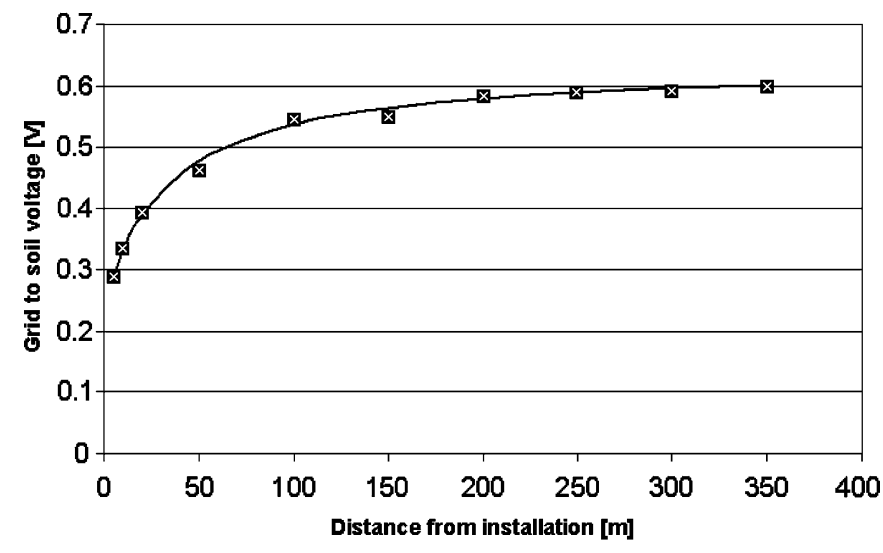

Fig. 7. Modified FOP test data compared with model response.

TABLE II

RESULTS OF ESTIMATION FOR CASE STUDY 2

\begin{tabular}{c|cc} 
File & Maximum Estimate & \%Error \\
\hline Original (18 points) & $1017 \mathrm{mV}$ & 51 \\
Modified (10 points) & $661 \mathrm{mV}$ & -2 \\
Original, Last Points & $620 \mathrm{mV}$ & -8
\end{tabular}

\section{CONCLUSION}

Based on the limited number of test cases examined no single method consistently produces an accurate or consistent method to estimate GPR from FOP data. However, it was found and shown that a set of four estimation methods can be used to estimate GPR and from that set an estimate for the GPR can be reasonably made. The estimation method is, however, subject to the quality of the data and the disturbances to the FOP response. If during the process of acquiring FOP results in the field disturbances can be identified, and affected data removed from the data set, then the accuracy of the estimations can be significantly increased. It was also shown that when the maximum estimate from the recommended optimal method set was used to provide the ultimate estimate, then the result was nominally conservative, in contrast to the normally nonconservative behavior of a traditional GPR estimate based on a FOR model.

\section{REFERENCES}

[1] D. J. Woodhouse and R. H. Middleton, "Assessment of analysis techniques used in determining grounding system potential rise from the fall of potential method," in Proc. IEEE Power Eng. Soc. Summer Meet., Seattle, WA, Jul. 2000.

[2] W. D. Carman and R. Hughes, "Safe earthing systems-part IV: earthing system analysis methods," in Proc. Electric Energy Conf., IEAust, Nov. 1990.

[3] IEEE Guide for Measuring Earth Resistivity, Ground Impedance, and Earth Surface Potentials of a Ground System, IEEE Std. 81-1983, 1983.

[4] IEEE Guide to Measurement of Impedance and Safety Characteristics of Large, Extended or Interconnected Grounding Systems, IEEE Std. 81.2-1991, Jun. 1992.

[5] IEEE Recommended Practice for Determining the Electric Power Station Ground Potential Rise and Induced Voltage From a Power Fault, IEEE Std. 367-1987, Apr. 1988.

[6] W. D. Carman, "A systematic earthing system design procedure employing probabilistic analysis of discrete solutions," Ph.D. dissertation, Univ. Newcastle, Department of Electrical Engineering, Newcastle, Australia, Feb. 2002. 
[7] J. M. Nahman, "Proximity effects on the ground fault current distribution within the earthing system formed by a substation and the associated transmission lines," Proc. Inst. Elect. Eng. C, vol. 135, no. 6, pp. 497-502, Nov. 1988.

[8] L. Ljung, System Identification-Theory for the User. Upper Saddle River, NJ: Prentice-Hall, 1987.

[9] W. Murray, Ed., Numerical Methods for Unconstrained Optimization. London, U.K.: Academic, 1972.

[10] R. H. Middleton and G. C. Goodwin, Digital Control and Estimation-A Unified Approach. Upper Saddle River, NJ: Prentice-Hall, 1990.

[11] R. W. Farebrother, Linear Least Squares Computations. New York: Marcel Dekker, 1988.

[12] E. D. Sunde, Earth Conduction Effects in Transmission Systems. London, U.K.: Dover, 1968.

[13] L. Sherwin, Explanatory Notes-Narromine 1:250000 Geological Sheet, New South Wales Department of Mineral Resources, 1996.

[14] J. Fortin, "Guide for measuring Hydro-Quebec's grounding system installations," Master of Applied Science Thesis, Report no. 85102, Electrical Engineering Department, Ecole Polytechnic, Univ. Montreal, Montreal, QC, Canada, Dec. 1985.

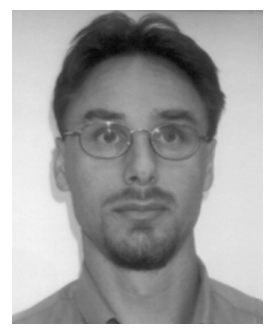

Darren J. Woodhouse was born in Maitland, Australia, on May 25, 1969. He received the B.E. (Hons.) and BMaths degrees and the Ph.D. degree in the area of earthing system testing from the University of Newcastle, Newcastle, Australia, in 1993, 1994, and 2004, respectively.

Currently, he is a Development Engineer with Safearth Engineered Solutions, a specialist unit of EnergyAustralia, Wallsend, Australia, a specialist engineering group focussed on earthing, including research and development. He joined EnergyAustralia (then Shortland Electricity), a state-owned energy services corporation with its head office in Sydney, Australia, in 1988, as a Cadet Engineer.

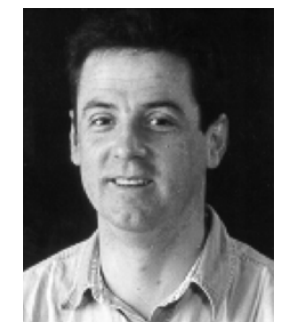

Richard H. Middleton (F'99) was born on December 10, 1961, in Newcastle Australia. He received the B.Sc., B.Eng. (Hons.), and Ph.D. degrees from the University of Newcastle, Australia, in 1983, 1984, and 1986, respectively.

Currently, he is a Panel Mmber of the Australian Research Council, a Professor in the Department of Electrical and Computer Engineering at the University of Newcastle, and Associate Director of the Centre for Integrated Dynamics and Control (A Commonwealth Special Research Centre). He was a Visiting Professor at the University of Illinois at Urbana-Champaign and at the University of Michigan, Ann Arbor. He was Head of the Department of Electrical and Computer Engineering at the University of Newcastle. His research interests include a broad range of control systems theory and applications.

Dr. Middleton was awarded the Australian Telecommunications and Electronics Research Board Outstanding Young Investigator award (national-annual award) in 1991. In 1994, he was awarded the Royal Society of New South Wales Edgeworth-David Medal (annual award for an outstanding young scientist). He is an Associate Editor for the IEEE TRANSACTIONS ON CONTROL System TECHNOLOGY and Automatica and was an Associate Editor of the IEEE TRANSACTIONS ON AUTOMATIC CONTROL. 